\title{
The Lateral Occipital Complex Is Activated by Melody with Accompaniment: Foreground and Background Segregation in Auditory Processing
}

\author{
Masayuki Satoh $^{1}$, Katsuhiko Takeda ${ }^{2}, K_{\text {Ken Nagata }}{ }^{3}$ Hidekazu Tomimoto ${ }^{4}$ \\ ${ }^{1}$ Department of Dementia Prevention and Therapeutics, Graduate School of Medicine, Mie University, Tsu, Japan \\ ${ }^{2}$ Department of Neurology, Mita Hospital, International Medicine and Welfare University, Tokyo, Japan \\ ${ }^{3}$ Department of Neurology, Research Institute for Brain and Blood Vessels, Akita, Japan \\ ${ }^{4}$ Department of Neurology, Graduate School of Medicine, Mie University, Tsu, Japan \\ E-mail: bruckner@clin.medic.mie-u.ac.jp \\ Received February 21, 2011; revised April 13, 2011; accepted May 15, 2011
}

\begin{abstract}
Objective: Most of the western music consists of a melody and an accompaniment. The melody is referred to as the foreground, with the accompaniment the background. In visual processing, the lateral occipital complex (LOC) is known to participate in foreground and background segregation. We investigated the role of LOC in music processing with use of positron emission tomography (PET). Method: Musically naïve subjects listened to unfamiliar novel melodies with (accompaniment condition) and without the accompaniment (melodic condition). Using a PET subtraction technique, we studied changes in regional cerebral blood flow (rCBF) during the accompaniment condition compared to the melodic condition. Results: The accompanyment condition was associated with bilateral increase of rCBF at the lateral and medial surfaces of both occipital lobes, medial parts of fusiform gyri, cingulate gyri, precentral gyri, insular cortices, and cerebellum. During the melodic condition, the activation at the anterior and posterior portions of the temporal lobes, medial surface of the frontal lobes, inferior frontal gyri, orbitofrontal cortices, inferior parietal lobules, and cerebellum was observed. Conclusions: The LOC participates in recognition of melody with accompaniment, a phenomenon that can be regarded as foreground and background segregation in auditory processing. The fusiform cortex which was known to participate in the color recognition might be activated by the recognition of flourish sounds by the accompaniment, compared to melodic condition. It is supposed that the LOC and fusiform cortex play similar functions beyond the difference of sensory modalities.
\end{abstract}

Keywords: Music, Positron Emission Tomography (PET), Melody, Accompaniment, Foreground and Background Segregation, Lateral Occipital Complex (LOC)

\section{Introduction}

The human visual cortex contains many areas whose functional characteristics, connectivity, and anatomy have been extensively documented [1]. Several studies have revealed the brain regions in association with recognition of the segregation between foreground and background $[1,2]$. Malach et al. reported a cortical region that responded more strongly when subjects passively viewed photographs of objects than when they viewed visual textures [3]. This region, which is located on the lateral bank of the fusiform gyrus extending ventrally and dor- sally, is named the lateral occipital complex (LOC) [2]. The LOC is thought to participate in the recognition of subjective contours involved in visual segregation between foreground and background, that is the figure as the foreground and the texture as the background [1]. Hasson et al. used a modified Rubin face-vase illusion (in which subjects perceived either a face or a vase) and reported that activation of the LOC correlated with times during which subjects reported they saw a face [4]. A study revealed that the LOC and cortex in its vicinity were activated more strongly when subjects touched objects than when they touched textures [5]. Thus, the LOC 
might constitute a neural substrate for convergence of multi-modal object representation [2].

Most songs in modern western music consist of a melody and accompaniment. Even if the melody and accompaniment are played by the same instrument (namely, sounds in the same timber) and even if they have a simple and almost equal rhythm (such as a chorale with the melody in the top part, supporting lines in the others, and a regular harmonic tread) [6], we can identify the melody from the accompaniment easily and effortlessly. The cognitive processing of that auditory identification is referred to as foreground-background processing [7]. The melody is referred to as the foreground, with the accompaniment the background. When we listen to music consists of a melody and an accompaniment, the segregation between them is automatically performed. If the LOC participates in foreground and background segregation not only in visual and somatosensory but also in auditory processing, it is likely that the LOC is activated when subjects listen to music consisting of a melody and accompaniment. In order to examine this hypothesis, we carried out a positron emission tomography (PET) activation study involving music perception. Musically naïve subjects listened to unfamiliar melodies with and without an accompaniment, and, using PET subtraction technique, the brain regions that were significantly activated by the accompaniment were identified.

\section{Subjects and Methods}

\subsection{Subjects}

Nine right-handed male volunteers (mean age 21.2 years; range 20 - 26), participated in the study. All were students at the Schools of Engineering or Mining, Akita University, and met criteria for Grison's second level of musical culture [8]. None had received any formal or private musical education, and none had any signs or history of neurological, cardiovascular or psychiatric disease. All subjects gave written informed consent after the purpose and procedure of the examination had been fully explained. The study was approved by the Ethics Committee of the Research Institute for Brain and Blood Vessels, Akita, Japan, and all experiments were conducted in accordance with the Declaration of Helsinki.

\subsection{Task Procedures}

The stimuli in this experiment were three new melodies that had been composed by one of the authors. All pieces were twenty-four bars in length and consisted of six phrases, or subdivisions, of a melodic line. All musical stimuli were played using a YAMAHA PSR-330 synthe- sizer set to piano-tone and recorded on a mini-disc. For each melody, following two tasks were performed: A) Melodic condition: as musical stimuli, melodies without the accompaniments were used. B) Accompaniment condition: For each melody, chords consisting of two tones and kept in harmony with the original melody were added. Two conditions were randomly represented. Subjects were instructed to listen to each melody, and PET measurements were obtained during this period (closely shown in the below). As behavior measures of performance, subjects were required to make a sign with the index finger of the right hand if they regarded some length of the tonal sequence as one phrase, that is a subdivision of a melodic line. All stimuli were presented randomly and binaurally via inset stereo-earphones.

The instruction for both conditions was as follows: Close your eyes. You will listen to a melody. If you regard some length of tonal sequence as one phrase, please make a sign with the index finger of your right hand.

\subsection{Positron Emission Tomography Measurements}

The protocol used in this study has been previously described in detail [9-11]. Briefly, PET data were acquired in 3-D acquisition mode using Headtome V (Shimazu, Kyoto, Japan). Scans were performed in a darkened room with subjects lying supine with eyes closed. Six CBF measurements were determined for each subject, three during the melodic condition and three during the accompaniment condition. Employing ${ }^{15} \mathrm{O}$ labeled water $\left(\mathrm{H}_{2}^{15} \mathrm{O}\right)$ intravenous bolus technique [12], emission data were collected for 90 seconds in each measurement following intravenous bolus injection of about $15 \mathrm{~mL}$ (40 mCi) $\mathrm{H}_{2}^{15} \mathrm{O}$. Each piece of music was started 15 seconds prior to data acquisition, repeated two times, and continued for about 120 seconds. Emission data were corrected for attenuation by acquiring 10 minutes of transmission data utilizing a ${ }^{68} \mathrm{Ge}$ orbiting rod source performed prior to the activation scans. A wash-out period of approximately 10 minutes was allowed between successive scans. For anatomic reference, all subjects underwent axial T1weighted imaging (T1WI) and T2-weighted imaging (T2WI) using a 1.5T magnetic resonance system (Vision, Siemens, Germany). T1WI (TR/TE = 665/14 ms) and T2WI (TR/TE = 3600/96 ms) were obtained using a slice thickness of $5 \mathrm{~mm}$ with an interslice gap of $1 \mathrm{~mm}$.

\subsection{Data Analysis}

PET data analysis was performed on a SGI Indy running IRIX 6.5 (Silicon Graphics, California), using an automated PET activation analysis package [13] composed of 
six main processing stages that has been previously described in detail [9-11]. The six main stages consisted of intra-subject co-registration; intra-subject normalization; automatic detection of the AC-PC line; detection of multiple stretching points and surface landmarks on intrasubject averaged image sets; inter-subject summation and statistical analyses; and superimposition of statistical results onto the stereotactic MRI. Deformation of individual brains to correspond with the standard atlas brain was achieved by spatially matching individual landmarks to the corresponding predefined standard surface landmarks and minimizing correlation coefficients of regional profile curves between the stretching centers. Activation foci were considered to be significantly activated if the corresponding $P$-value was less than a pre-determined threshold $(P<0.01$, Bonferroni correction for multiple comparisons, 117,882 pixels). Anatomical identification of activation foci was achieved by referring the stereotactic coordinates of the peak activated pixels to the standard Talairach brain atlas [14].

\section{Results}

For behavioral measures of performance for the melody and accompaniment conditions, the mean correct response was almost $93 \% \pm 6.3$ (mean \pm standard deviation) for both conditions. We conclude that subjects performed experimental tasks reasonably well. The results of subtractions in terms of significant regions activated during each condition are given in Tables $\mathbf{1}$ and $\mathbf{2}$ and Figures $\mathbf{1}$ and 2 . The regions activated during the accompaniment condition, but not during the melodic condition are listed in Table 1 together with stereotactic coordinates based on the brain atlas of Talairach and Tournoux [14]. These results show areas of relative blood flow changes that emphasize differences between the two conditions and minimize areas that are common to both conditions. Significant increases of relative cortical blood flow were found in the lateral and medial surfaces of both occipital lobes, medial parts of fusiform gyri, cingulate gyri, precentral gyri, insular cortices, and cerebellum (Figure 1).

Table 1. Regions showing significant changes in rCBF during the accompaniment condition.

\begin{tabular}{|c|c|c|c|c|c|c|}
\hline \multirow{2}{*}{ Anatomical structures } & & \multirow{2}{*}{ Brodmann area } & \multicolumn{3}{|c|}{ Talairach coordinate } & \multirow{2}{*}{ z-score } \\
\hline & & & $\mathrm{x}$ & $\mathrm{y}$ & $\mathrm{z}$ & \\
\hline \multirow[t]{3}{*}{ Lateral surface of occipital lobe } & & $18 / 19$ & & & & \\
\hline & $\mathrm{L}$ & & -26 & -78 & 20 & 2.74 \\
\hline & $\mathrm{R}$ & & 37 & -71 & -7 & 5.76 \\
\hline \multirow[t]{3}{*}{ Fusiform gyrus } & & $19 / 37$ & & & & \\
\hline & $\mathrm{L}$ & & -35 & -60 & -7 & 3.65 \\
\hline & $\mathrm{R}$ & & 39 & -42 & -16 & 3.54 \\
\hline \multicolumn{7}{|l|}{ Medial surface of occipital lobe } \\
\hline & $\mathrm{L}$ & 17 & -19 & -87 & 2 & 4.79 \\
\hline & $\mathrm{R}$ & 18 & 17 & -78 & 27 & 3.92 \\
\hline \multirow[t]{3}{*}{ Cingulate gyrus } & & $24 / 32$ & & & & \\
\hline & $\mathrm{L}$ & & -12 & -13 & 38 & 2.75 \\
\hline & $\mathrm{R}$ & & 8 & -15 & 40 & 2.45 \\
\hline \multirow[t]{3}{*}{ Precentral gyrus } & & 6 & & & & \\
\hline & $\mathrm{L}$ & & -53 & -4 & 27 & 3.8 \\
\hline & $\mathrm{R}$ & & 17 & -31 & 61 & 2.98 \\
\hline \multirow[t]{3}{*}{ Inslar cortex } & & 41 & & & & \\
\hline & $\mathrm{L}$ & & -33 & -19 & 14 & 3.1 \\
\hline & $\mathrm{R}$ & & 37 & -22 & 20 & 4.1 \\
\hline \multicolumn{7}{|l|}{ Cerebellum } \\
\hline & $\mathrm{L}$ & & -24 & -58 & -45 & 3.37 \\
\hline & $\mathrm{R}$ & & 15 & -64 & -7 & 3.55 \\
\hline
\end{tabular}

Coordinates $\mathrm{x}, \mathrm{y}, \mathrm{z}$ are in millimetres corresponding to the atlas of Talairach and Tournoux. The x-coordinate refers to medial-lateral position relative to midline (negative = left); y-coordinate anterior-posterior position relative to the anterior commissure (positive = anterior); z-coordinate superior-inferior position relative to the anterior commissure-posterior commissure line (positive = superior). $\mathrm{Z}$ refers to the $\mathrm{z}$-score of the maximum pixel in the region. $\mathrm{L}$ and $\mathrm{R}$ refer to the left and right hemisphere, respectively. 
Table 2. Regions showing significant changes in rCBF during the melodic condition.

\begin{tabular}{|c|c|c|c|c|c|c|}
\hline \multirow{2}{*}{ Anatomical structures } & & \multirow{2}{*}{ Brodmann area } & \multicolumn{3}{|c|}{ Talairach coordinate } & \multirow{2}{*}{ Z } \\
\hline & & & $\mathrm{x}$ & $\mathrm{y}$ & $\mathrm{z}$ & \\
\hline \multicolumn{7}{|l|}{ Anterior portion of temporal lobe } \\
\hline & $\mathrm{L}$ & 38 & -26 & 14 & -29 & 3.71 \\
\hline & $\mathrm{R}$ & 21 & 48 & -6 & -9 & 3.17 \\
\hline & & 38 & 26 & 8 & -38 & 4.28 \\
\hline \multirow[t]{3}{*}{ Posterior portion of temporal lobe } & & 21 & & & & \\
\hline & $\mathrm{L}$ & & -55 & v31 & -4 & 2.88 \\
\hline & $\mathrm{R}$ & & 57 & -40 & -2 & 4.39 \\
\hline \multirow[t]{5}{*}{ Medial surface of frontal lobe } & & $8 / 9$ & & & & \\
\hline & $\mathrm{L}$ & & -1 & 17 & 61 & 4.64 \\
\hline & & & -10 & 48 & 47 & 2.99 \\
\hline & $\mathrm{R}$ & & 8 & 44 & 50 & 4.19 \\
\hline & & & 28 & 46 & 32 & 3.23 \\
\hline \multirow[t]{2}{*}{ Inferior forntal gyrus } & & 45 & & & & \\
\hline & $\mathrm{R}$ & & 48 & 19 & 11 & 3.58 \\
\hline \multirow[t]{3}{*}{ Orbitofrontal cortex } & & $11 / 47$ & & & & \\
\hline & $\mathrm{L}$ & & -12 & 41 & -22 & 3.39 \\
\hline & $\mathrm{R}$ & & 28 & 21 & -7 & 3.31 \\
\hline \multicolumn{7}{|l|}{ Inferior parietal lobule } \\
\hline & $\mathrm{L}$ & 40 & -51 & -46 & 45 & 2.43 \\
\hline & $\mathrm{R}$ & 40 & 55 & -37 & 27 & 3.55 \\
\hline & & 39 & 51 & -58 & 36 & 2.95 \\
\hline \multicolumn{7}{|l|}{ Cerebellum } \\
\hline & $\mathrm{L}$ & & -8 & -73 & -43 & 5.03 \\
\hline & $\mathrm{R}$ & & 10 & -82 & -32 & 3.36 \\
\hline
\end{tabular}

Details as for Table 1.

The melodic condition produced significantly greater activation bilaterally than the accompaniment task in the anterior and posterior portions of the temporal lobes, medial surface of the frontal lobes, inferior frontal gyri, orbitofrontal cortices, inferior parietal lobules, and cerebellum (Figure 2, Table 2).

\section{Discussion}

\subsection{Lateral Occipital Complex (LOC) Participation in the Segregation between Melody and Accompaniment}

For the current study, we suppose that the LOC has a relationship with the segregation between foreground and background not only in visual and somatosensory, but also in auditory processing. In our experiment, the lateral surface of the occipital lobe which contained the regions of the LOC and its vicinity was activated during listening to the melody with accompaniment, but not to the melody without accompaniment. In reported literatures, the activation of this region was also observed in listening to a popular song with an accompaniment of Schmithorst's experiment [15]. Using functional magnetic resonance imaging (MRI), Schmithorst investigated the activated brain regions when subjects listened to popular melodies with or without harmonized accompaniment [15]. Compared with the latter condition, the former (namely, melody with accompaniment) bilaterally activated the inferior occipital and fusiform gyri, which seemed to contain the regions of the LOC and its vicinity. These regions were similar to the brain regions activated 


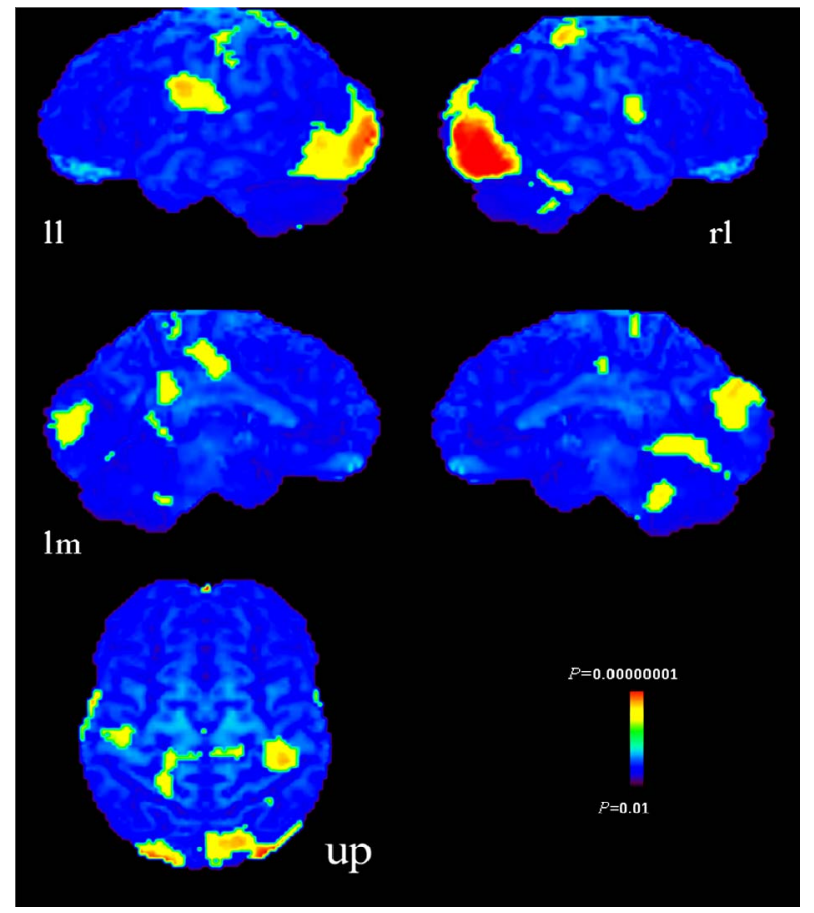

Figure 1. Activation maps for the subtraction of the accompaniment versus the melodic condition. Areas of significant activation $(p<0.001)$ are superimposed onto the surface maps of the averaged MR imaging of the brains of nine subjects. The lateral and medial surface of both occipital lobes, medial parts of fusiform gyri, cingulate gyri, precentral gyri, insular cortices, and cerebellum were remarkably activated. Lateral surface of left hemisphere (II); medial surface of left hemisphere (lm); lateral surface of right hemisphere (rl); medial surface of right hemisphere (rm); upper surface (up). The left side of the bottom-image shows the left side of the brain.

during our accompaniment task. They did not denote the accurate function of these brain regions, and not mention cognitive processing of the segregation between melody and accompaniment. Using ${ }^{15} \mathrm{O}$-water PET, Brown et al. [16] studied the song system of human brain: singing repetitions of novel melodies and singing harmonizations with novel melodies. Comparing the rest condition, the latter task showed the bilateral activation of frontal operculums, anterior portion of temporal lobes, superior temporal gyri, and right ventral occipito-temporal cortex which belonged to the LOC. The authors presented the activation of ventral occipito-temporal cortex only on their figure, and they did not mention it on the table and in discussion. From the results of Schmithorst's [15], Brown's [16], and the present study, we may say that the LOC might participate in recognition of melody as the foreground and the accompaniment as the background. To the best of our knowledge, there was no case report which described the impairment of the segregation between melody and accompaniment due to the lesion of

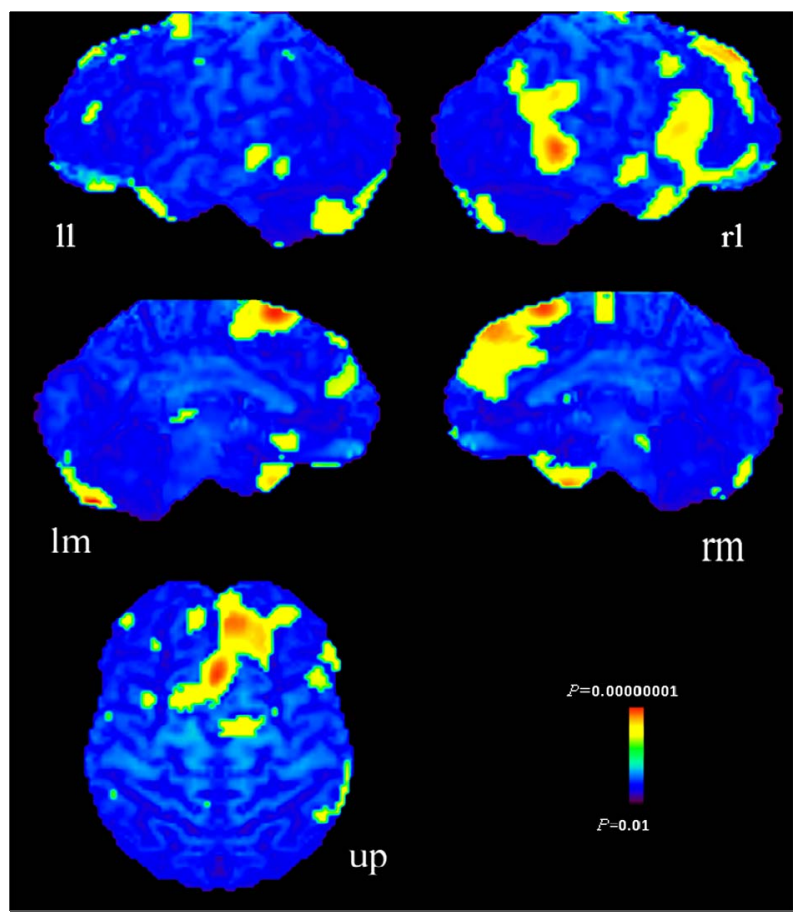

Figure 2. Activation maps for the subtraction of the melodic versus accompaniment condition. Areas of significant activation $(p<0.001)$ are superimposed onto the surface maps of the averaged MR imaging of the brains of nine subjects. Anterior and posterior portions of the temporal lobes, medial surface of the frontal lobes, inferior frontal gyri, orbitofrontal cortices, inferior parietal lobules, and cerebellum were bilaterally activated. Details as for Figure 1.

the LOC. Such case studies will ascertain the multi-modal function of the LOC of foreground and background segregation.

Someone may ask, "Is it possible that the LOC participates in perception of harmony?" The brain regions which participate in the perception of harmony remained unclear, but, based on the results of a previous PET activation study [10], we can reply in the negative. That study evaluated which brain regions were activated when subjects listened to the soprano part or harmony of new identical musical pieces [10]. When listening to the soprano part was compared with listening to the harmony, there was significantly greater activation bilaterally in the lateral occipital lobes, which contain the LOC. While listening to the harmony, the anterior portions of both temporal lobes were activated, but not the LOC. So, we may say that during listening to the soprano part the LOC played a role in recognition of melody as the foreground and accompaniment of the harmony as the background, results that are consistent with those of the present study. As closely discussed below, the comparison between the accompaniment and a passive resting condition might be needed to settle this problem. 
The fusiform cortex is known to participate in color recognition from case [17] and PET activation studies [18]. The sound of the melody and accompaniment is richer, in other words flourisher, than that of the melody without accompaniment. The fusiform cortex might be activated by recognition of rich sounds that were represented by the accompaniment condition of our present and Schmithorst's [15] study. We may say that, in the human brain, some neural substrates of association cortices, for example the LOC and the fusiform cortex, carried out the similar functions beyond the difference of sensory modalities.

The neurofunctional significance of the insular cortex remains unsettled. The insular cortex has connection with auditory and visual association and somatosensory cortices. The insular cortex may have an important role in multimodal convergence of sensory input [19] and the conscious awareness of feeling emotions [20,21]. In this experiment, this region might participate in the reference of auditory information to the visual cognitive system via the connection to the auditory and visual association cortices, and in the emotion which was induced by the flourish sound by the harmony of the accompaniment.

Activation of cingulate gyri and cerebellum has been observed in PET activation studies regarding music processing in the brain $[9,10,22,23]$. To date, anatomical, physiological, and functional neuroimaging studies have suggested that the cerebellum participates in the organization of higher cognitive functions [24] including music processing [25]. These brain regions might be activated in relation to mental processes to anticipate a new learned task and involve in trying to identify musical stimuli.

\subsection{Activated Brain Regions during the Melodic Condition}

Since the accompaniment condition also contained melody, the significance of the activated regions in the melodic condition can not be explained by the presence or absence of melody perception during performances. In the accompaniment condition, the subjects might perform some different kinds of cognitive processing compared to the melodic condition: listened to sound of music as a whole and segregated the melody and its accompaniment from that sound. So, it is possible that the degree of melody perception can be different between two conditions. Under such a limitation, we tried to discuss below the function of brain regions which activated during the melodic condition.

We hypothesized that during the melodic condition, the temporal and frontal lobes would participate in perception of melody and auditory imagery. A previous report has pointed out that the anterior portions of the temporal lobes are activated when subjects listen to familiar melodies [11]. In another study of patients who underwent temporal lobectomy for treatment of epilepsy, patients with right or left lobectomy had a deficit in recognition of familiar or unfamiliar melodies [26-28]. In our present experiment, too, the anterior portions of the temporal lobes might have been activated by melody perception.

We may say that the frontal and posterior portions of the temporal lobes have a relationship with auditory imagery processing. Using PET, Halpern and Zatorre reported that the frontal and posterior superior temporal regions and medial frontal cortices were activated by auditory imagery for familiar tunes [29]. Several neuroimaging studies that used functional MRI and MEG have supported this opinion [30,31]. Thus, activation of posterior portions of temporal lobes and frontal regions in our melodic condition might have been caused by the generation of melodic imagery.

The inferior parietal lobules with frontal regions might be activated by attention [32-34]. As for activation of the cerebellum, its exact function remains unclear. However, the pattern of activation was different between the melodic and accompaniment conditions. Our results suggest the anatomical localization of higher cognitive functions within the cerebellum.

\subsection{The Limitations}

This study has some limitations. First, the comparison of rCBF between two conditions and the resting state was not carried out, so it could not be concluded whether the results of the study were due to the decrease or the increase of rCBF in LOC during the melodic or accompaniment condition, respectively. It is well known that, in functional neuroimaging data, we often observe activity decreases when the task condition was compared with passive resting state. The presence of such decreases implied the existence of a default mode of the brain which might participate in the maintenance of information for interpreting, responding to and predicting environmental demands [35]. But, some researchers suggested that resting scans were likely to be confounded by group differences in vascular factors or rCBF coupling, and the poorly defined cognitive nature of "rest" offered little that enabled us to distinguish specific psychological and physiological processing [36]. We did not know the literature which reported the decrease of $\mathrm{rCBF}$ at the brain regions shown in the present study during performing musical tasks. Much still remains to be done about the interpretation of meaning of resting state in activation study.

Second, the subjects were young and male, so it is an 
unsettled question whether the results would be consistent across different age and gender groups.

And third, the activated brain region during the melodic condition could not be completely determined, since the accompaniment condition also contained the melody. The difference of the activation might be caused by the difference degree of the cognitive processing of melody perception between two conditions.

\section{Conclusions}

The LOC was activated when subjects listened to melody with accompaniment. It has been postulated that the LOC participates in the segregation between foreground and background in visual, somatosensory, and auditory processing. The fusiform cortex might be activated by recognition of flourish sound in a manner that is similar with visual processing. During the melodic condition, temporal and frontal lobes were mainly activated. Those regions have a relationship with the perception of melody and auditory imagery processing. Additional experiments, especially with passive resting condition, will be necessary to completely determine the significance of the activated brain regions in the present study, and how the same neural substrates carry out the similar functions beyond the difference of sensory modalities.

\section{References}

[1] K. Grill-Spector, T. Kushnir, S. Edelman, V. Itzchak and R. Malach, "Cue-Invariant Activation in Object-Related Areas of the Human Occipital Lobe," Neuron, Vol. 21, No. 1, 1998, pp. 191-202. doi:10.1016/S0896-6273(00)80526-7

[2] K. Grill-Spector, Z. Kourtzi and N. Kanwisher, "The Lateral Occipital Complex and Its Role in Object Recognition," Vision Research, Vol. 41, No. 10-11, 2001, pp. 1409-1422. doi:10.1016/S0042-6989(01)00073-6

[3] R. Malach, J. B. Reppas, R. R. Benson, K. K. K. Kwong, H. Jiang, W. A. Kennedy, P. J. Ledden, T. J. Brady, B. R. Rosen and R. B. H. Tootell, “Object-Related Activity Revealed by Functional Magnetic Resonance Imaging in Human Occipital Cortex," Proceedings of the National Academy of Sciences of the USA, Vol. 92, No. 18, 1995, pp. 8135-8139. doi:10.1073/pnas.92.18.8135

[4] U. Hasson, T. Hendler, D. B. Bashat and R. Malach, "Vase or Face? Neural Correlate of Shape-Selective Grouping Processes in the Human Brain,” Journal of Cognitive Neuroscience, Vol. 13, No. 6, 2001, pp. 744-753. doi:10.1162/08989290152541412

[5] A. Amedi, R. Malach, T. Hendler, P. Sharon and E. Zohary, "Visuo-Haptic Object-Related Activation in the Ventral Visual Pathway,” Nature Neuroscience, Vol. 4, No. 3, 2001, pp. 324-330. doi:10.1038/85201

[6] S. Sadie, "The Grove Concise Dictionary of Music,"
Macmillan, London, 1994.

[7] B. Snyder, "Melody," In: B. Snyder, Ed., Music and Memory, MIT Press, Cambridge, 2000, pp. 135-158.

[8] B. Grison, "Une Etude sur les Alterations Musicales au Cours des Lesions Hemispheriques,” Thesis, Paris, 1972. Cited by A. L. Benton, “The Amusias,” In: M. Critchley and R. A. Henson, Eds., Music and the Brain, William Heinemann Medical Books Limited, London, 1977, pp. 378-397.

[9] M. Satoh, K. Takeda, K. Nagata, J. Hatazawa and S. Kuzuhara, "Activated Brain Regions in Musicians During An Ensemble: A PET Study,” Cognitive Brain Research, Vol. 12, No. 1, 2001, pp. 191-108. doi:10.1016/S0926-6410(01)00044-1

[10] M. Satoh, K. Takeda, K. Nagata, J. Hatazawa, and S. Kuzuhara, "The Anterior Portion of the Bilateral Temporal Lobes Participates in Music Perception: A Positron Emission Tomography Study,” American Journal of Neuroradiology, Vol. 24, No. 9, 2003, pp. 1843-1848.

[11] M. Satoh, K. Takeda, K. Nagata, E. Shimosegawa and S. Kuzuhara, "Positron Emission Tomography of Brain Regions Activated by Recognition of Familiar Music," American Journal of Neuroradiologyl, Vol. 27, No.5 , 2006, pp. 1101-1106.

[12] I. Kanno, H. Iida, S. Miura, M. Murakami, K. Takahashi, H. Sasaki, A. Inugami, F. Shishido and K. Uemura, "A System for Cerebral Blood Flow Measurement Using an $\mathrm{H}_{2}{ }^{15} \mathrm{O}$ Autoradiographic Method and Positron Emission Tomography," Journal of Cerebral Blood Flow \& Metabolism, Vol. 7, 1987, pp. 143-153. doi:10.1038/jcbfm.1987.37

[13] S. Minoshima, R. A. Koeppe, J. A. Fessler, M. A. Mintum, K. L. Berger, S. F. Taylor and D. E. Kuhl, "Integrated and Automated Data Analysis Method for Neuronal Activation Studies Using [O-15] Water PET,” In: K. Uemura, N. A. Lasen, T. Jones and I. Kannno, Eds., Quantification of Brain Function, Tracer Kinetics and Image Analysis in Brain PET, Excerpta Medica/Elsevier, Amsterdam, 1993, pp. 409-417.

[14] J. Talairach and P. Tournoux, "Co-Planar Stereotaxic Atlas of the Human Brain,” Thieme, New York, 1988.

[15] V. J. Schmithorst, "Separate Cortical Networks Involved in Music Perception: Preliminary Functional MRI Evidence for Modularity of Music Processing," Neuroimage, Vol. 25, No. 2, 2005, pp. 444-451. doi:10.1016/j.neuroimage.2004.12.006

[16] S. Brown, M. J. Martinez, D. A. Hodges, P. T. Fox and L. M. Parsons, "The Song System of the Human Brain," Cognitive Brain Research, Vol. 20, No. 3, 2004, pp. 363375. doi:10.1016/j.cogbrainres.2004.03.016

[17] A. R. Damasio, T. Yamada, H. Damasio, J. Corbett and J. McKee, "Central Achromatopsia: Behavioral, Anatomic, and Physiological Aspects,” Neurology, Vol. 30, 1980, pp. 1064-1071.

[18] M. Corbetta, F. M. Miezin, S. Dobmeyer, G. L. Shulman and S. E. Petersen, "Attentional Modulation of Neural Processing of Shape, Color, and Velocity in Humans," 
Science, Vol. 248, No. 4962, 1990, pp. 1556-1559. doi:10.1126/science.2360050

[19] M. M. Mesulam, and E. J. Mufson, "The Insula of Reil in Man and Monkey: Architectonics, Connectivity, and Function,” In: A. Peters and E. G. Jones, Eds., Cerebral Cortex, Plenum Press, New York, 1985, pp. 179-226.

[20] A. D. Craig, "How Do You Feel? Interoception: The Sense of the Physiological Condition of the Body," Nature Reviews Neuroscience, Vol. 3, No. 8, 2002, pp. 655666.

[21] E. L. Johnsen, D. Tranel, S. Lutgendorf and R. Adolph, "A Neuroanatomical Dissociation for Emotion Induced by Music," International Journal of Psychophysiology, Vol. 72, No. 1, 2009, pp. 24-33. doi:10.1016/j.ijpsycho.2008.03.011

[22] A. J. Blood and R. J. Zatorre, "Intensely Pleasurable Responses to Music Correlate with Activity in Brain Regions Implicated in Reward and Emotion,” Proceedings of the National Academy of Sciences of the USA, Vol. 98, No. 20, 2001, pp. 11818-11823. doi:10.1073/pnas.191355898

[23] P. Janata, B. Tillmann and J. Bharucha, "Listening to Polyphonic Music Recruits Domain-General Attention and Working Memory Circuits," Cognitive, Affective \& Behavioral Neuroscience, Vol. 2, No. 2, 2002, pp. 121140. doi:10.3758/CABN.2.2.121

[24] J. D. Schmahmann and J. C. Sherman, "The Cerebellar Cognitive Affective Syndrome,” Brain, Vol. 121, No. 4, 1998, pp. 561-579. doi:10.1093/brain/121.4.561

[25] L. M. Parsons, "Exploring the Functional Neuroanatomy of Music Performance, Perception, and Comprehension,” In: I. Peretz and R. Zatorre Eds., The Cognitive Neuroscience of Music, Oxford University Press, New York, 2003, pp. 247-268.

[26] B. Milner, "Laterality Effects in Audition," In: V. B. Mountcastle, Ed., Interhemispheric Relations and Cerebral Dominance, Johns Hopkins University Press, Baltimore, 1962, pp. 177-195.

[27] R. J. Zatorre, "Discrimination and Recognition of Tonal Melodies after Unilateral Cerebral Excisions,” Neuropsychologia, Vol. 23, No. 1, 1985, pp. 31-41.

\section{doi:10.1016/0028-3932(85)90041-7}

[28] M. Dennis and T. Hopyan, "Rhythm and Melody in Children and Adolescents after Left or Right Temporal Lobectomy,” Brain and Cognition, Vol. 47, No. 3, 2001, pp. 461-469. doi:10.1006/brcg.2001.1322

[29] A. R. Halpern and R. J. Zatorre, "When That Tune Runs Through Your Head: A PET Investigation of Auditory Imagery for Familiar Melodies,” Cerebral Cortex, Vol. 9, No. 7, 1999, pp. 697-704. doi:10.1093/cercor/9.7.697

[30] S. S. Yoo, C. U. Lee and B. G. Choi, "Human Brain Mapping of Auditory Imagery: Event-Related Functional MRI Study,” Neuroreport, Vol. 12, 2001, pp. 3045-3049. doi:10.1097/00001756-200110080-00013

[31] M. Schurmann, T. Raij, N. Fujiki and R. Hari, "Mind's ear in a Musician: Where and When in the Brain," Neuroimage, Vol. 16, No. 2, 2002, pp. 434-440. doi:10.1006/nimg.2002.1098

[32] M. Corbetta, S. Miezin, S. Dobmeyer, G. L. Shulman and S. E. Petersen, "Selective and Divided Attention during Visual Discrimination of Shape, Color, and Speed: Functional Anatomy by Positron Emission Tomography," Journal of Neuroscience, Vol. 11, No. 8, 1991, pp. 23832402.

[33] B. Kolb, I. Q. Whishaw, “Attention, Imagery, and Consciousness,” In: B. Kolb and I. Q. Whishaw Eds., Fundamental of Human Neuropsychology, W. H. Freeman, New York, 1996, pp. 465-476.

[34] V. Poghosyan, T. Shibata and A. A. Ioannides, "Effects of Attention and Arousal on Early Responses in Striate Cortex,” European Journal of Neuroscience, Vol. 22, No. 1, 2005, pp. 225-234. doi:10.1111/j.1460-9568.2005.04181.x

[35] M. E. Raichle and A. Z. Snyder, "A Default Mode of Brain Function: A Brief History of an Evolving Idea," Neuroimage, Vol. 37, No. 4, 2007, pp. 1083-1090. doi:10.1016/j.neuroimage.2007.02.041

[36] A. M. Morcom and P. C. Fletcher, "Cognitive Neuroscience: The Case for Design Rather than Default," Neuroimage, Vol. 37, No. 4, 2007, pp. 1097-1099. doi:10.1016/j.neuroimage.2007.07.018 